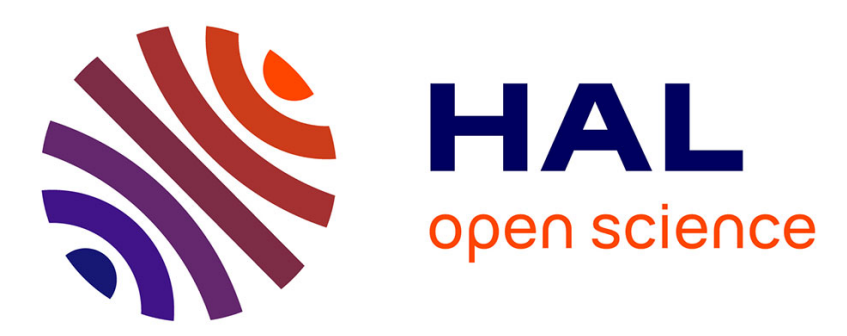

\title{
LOCALISATION DE SOURCES PAR INTENSIMÉTRIE TRIDIMENSIONNELLE
}

\author{
J. Rebillat, J. Patrat
}

\section{To cite this version:}

J. Rebillat, J. Patrat. LOCALISATION DE SOURCES PAR INTENSIMÉTRIE TRIDIMENSIONNELLE. Journal de Physique IV Proceedings, 1992, 02 (C1), pp.C1-435-C1-439. 10.1051/jp4:1992193 . jpa-00251265

\section{HAL Id: jpa-00251265 https://hal.science/jpa-00251265}

Submitted on 1 Jan 1992

HAL is a multi-disciplinary open access archive for the deposit and dissemination of scientific research documents, whether they are published or not. The documents may come from teaching and research institutions in France or abroad, or from public or private research centers.
L'archive ouverte pluridisciplinaire HAL, est destinée au dépôt et à la diffusion de documents scientifiques de niveau recherche, publiés ou non, émanant des établissements d'enseignement et de recherche français ou étrangers, des laboratoires publics ou privés. 


\title{
LOCALISATION DE SOURCES PAR INTENSIMETRIE TRIDIMENSIONNELLE
}

\author{
J.C. REBILLAT et J.C. PATRAT \\ Laboratoire d'Etudes Aérodynamiques (L.E.A.), C.E.A.T., 43 route de l'Aérodrome, \\ F-86000 Poitiers, France
}

\begin{abstract}
RESUME : Nous proposons une nouvelle méthode de localisation de sources acoustiques, utilisant la sonde intensimetrique tridimensionnelle mise au point au C.E.A.T. Dans le cas de sources placées sur une surface ou une ligne connue, une mesure unique est possible. Cependant 11 existe un blais de mesure. Des mesures en plusieurs points permettent de supprimer ce biais et donnent des informations sur 1 'etendue spatiale de la source.

ABSTRACT: The aim of this paper is a new method to localise real acoustical sources lying on knowned surfaces or axes, using C.E.A.T. new 3D acoustical intensimetry probe.A blas measurement exists when doing only one measurement. This error is lower than a given calculated value. Multipoints measurements allow biasless determination and give an approached value of the length of the source.
\end{abstract}

\section{I : INTRODUCTION.}

Les études de bruit de jets supersoniques, en vue de localisation des sources en particulier, en liaison avec les problèmes vibro-acoustiques des lanceurs, conservent une importance actuelle [1 et 2].

$\mathrm{Au}$ L.E.A. [3], nous avons mis au point une méthode de détermination du vecteur Intensité Acoustique $I$, grâce à une sonde sphérique. Dans le cas d'une source monopolaire isotrope, la direction de I indique celle de la source. Si la source est sur un axe ou une surface simple connue, une seule détermination de $I$ permet une localisation spatiale. Deux mesures sont nécessaires dans le cas le plus général.

Cette méthode parait utilisable pour localiser les sources acoustiques dans un jet, dans l'hypothèse où elles sont sur l'axe de celui-ci. Mais les sources ont une étendue spatiale qui ne permet pas l'hypothèse monopolaire.

Nous montrons que, dans ce cas, des erreurs de biais apparaissent. Une borne supérieure de cette erreur est calculée, en utilisant une modélisation numérique plane de l'Intensité Acoustique de $\mathbf{N}$ monopôles [4].

\section{II : BIAIS DE MESURE}

Soit, figure 1, une source à symétrie de révolution autour de l'axe ox et de centre $X$. L'Intensité I mesurée en $M$, détermine sur cet axe un centre apparent $X^{\prime}$.

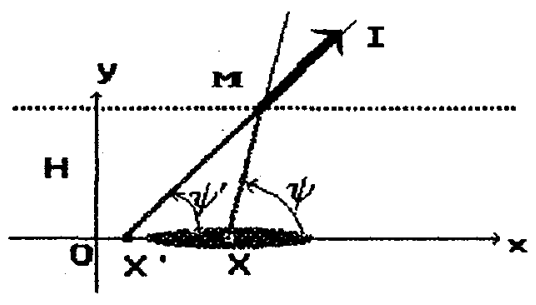
L'éloignement $\Delta \mathrm{X}$ de ces deux centres caractérise l'erreur de localisation

$$
\begin{aligned}
& \Delta x=\overrightarrow{X^{\prime} X}=\frac{-\sin \left(\psi^{\prime}-\psi\right)}{\sin \psi \sin \psi^{\prime}} H \\
& \psi=(\overrightarrow{X X}, \overrightarrow{X M}) \psi^{\prime}=\left(\overrightarrow{X^{\prime}} \vec{X}, \overrightarrow{X^{\prime} M}\right) \\
& \text { si } \Delta \psi=\psi^{\prime}-\psi \text { est petit alors } \Delta X \simeq-\frac{\Delta \psi}{\sin ^{2} \psi} H
\end{aligned}
$$

Figure 1 : Biais de mesure

Soit $\mathrm{L}$ une longueur caractéristique du problème physique, pour avoir des grandeurs géométriques adimensionelles, on pose $x=X / L, \varepsilon=\Delta X / L, h=H / L, r=R / L \ldots$. et $k=k L$. 
III : MODELE DE RAYONNEMENT ACOUSTIQUE DE SOURCES PONCTUELLES [4]

Soit $N$ monopóles dans un plan, aux points $\left(r_{i}, \theta_{i}\right)$. Le pôle $i$ est caractérisé par son intensité réduite $a(\omega)$, une phase $\eta_{i}$, ainsi que par la fonction de cohérence entre deux pôles $i$ et $j: \gamma_{1 j}^{2}(\omega)$. Avec $A_{0}$ une intensité de référence, on calcule, au point $M(r, \theta)$, une intensité réduite, colinéaire avec le vecteur Intensité acoustique.. Le vecteur $I$ est donné par ses deux composantes radiale et tangentielle :

$$
\begin{aligned}
& I_{R}(r, \omega)=\sum_{i} a_{i}^{2} \frac{r^{2}}{r_{i}^{2}} \frac{\partial r}{\partial r}+\sum_{i, j_{i \neq j}} r_{i j} a_{j} \frac{r^{2}}{r_{i} r} \frac{\partial r}{\partial r}\left(\cos \phi_{i j}+\frac{1}{k r} \sin \phi_{i j}\right) \\
& \phi_{i j}=k\left(r_{i}-r_{j}\right)-\left(\eta_{1}-n_{j}\right)
\end{aligned}
$$

La composante tangentielle $I_{\mathrm{T}}$ s'obtient en remplaçant $\left.\partial \mathrm{r}\right\} / \partial \mathrm{r}$ par $(1 / \mathrm{r})$. $\partial \mathrm{r} \mathrm{J} / \partial \theta$ La déviation angulaire est obtenue à partir de l'arc tangente de $I_{\mathrm{T}} / I_{\mathrm{R}}$.

$$
\text { IV : DIPOLE. }
$$

Le cas théorique le plus simple est le dipôle centré à l'origine. Dés lors l'incidence $\theta$ s'identifie avec $\psi$. Les pôles sont $P_{1}(1,0)$ et $P_{2}(1, \pi)$, en opposition de phase. Soit la longueur effective L égale à la $1 / 2$ distance entre les pôles. Pour $r$ grand :

$$
r_{1} \simeq r_{2} \simeq r \text { et } r_{1}-r_{2} \simeq 2 \operatorname{Cos} \theta \text { quand } r \rightarrow \infty
$$

Pour une cohérence différente de 1 , les équations 3 conduisent à :

$$
I_{\mathrm{R}} \simeq 2\left(1-\gamma_{12}\right) \quad I_{\mathrm{T}} \simeq \frac{-6}{\mathrm{r}^{2}}\left(1-\gamma_{12}\right) \text { sinecose pour } \mathrm{r} \rightarrow \infty
$$

Comme $I_{\mathrm{T}} / I_{\mathrm{R}}$ est petit, en utilisant la formule approchée $(2)$ :

$$
\varepsilon \simeq(1,5 \sin 2 \theta) / \mathrm{h}
$$

L'erreur est maximale pour l'incidence $\theta=\pi / 4$. Elle est nulle à $l$ 'aplomb de la source et très loin en amont et en aval. A cause des dispersions de mesure, cette dernière propriété n'est pas exploitable en pratique.

$\varepsilon$ est inversement proportionnelle à h. Ceci nous conduit à proposer de caractériser le biais de localisation par une erreur standard $\mu=\varepsilon . h$. En effet pour un dipôle $\mu$ est indépendant de $h$.

$$
\text { ERREUR STANDARD } \mu=\varepsilon \mathbf{h}=\frac{\Delta \mathrm{X}}{\mathrm{L}} \frac{\mathrm{H}}{\mathrm{L}}
$$

Dans le cas d'un dipôle l'équation 6 permet de calculer une valeur maximale, erreur standard maximale $\mu_{M}$ :

$$
\frac{\text { BORNE SUPERIEURE } \mu_{\mathrm{m}}=1,5}{\mathrm{~V}: \text { SOURCE ETENDUE LE LONG D'UN AXE }}
$$

\section{V.1 : Modélisation}

Nous proposons de modéliser des sources supposées placées sur l'axe d'un jet (figure 1) par un ensemble de $N=2 M+1$ monopôles aux points de coordonnées cartésiennes : $x_{i}$ et $y_{1}=0$. La source centrale $M$ est à l'origine. Les hypothèses faites sont :

H1 : les pôles sont en phase.

H2 : la répartition des intensités polaires ou des cohérences est gaussienne, de la forme :

$$
\operatorname{Exp}\left(\frac{j^{2}}{\alpha M^{2}}\right) \text { avec } \alpha=4,3429 / \beta
$$


Pour calculer $a_{i}, j=|i-(M+1)|$ avec $\beta$ l'affaiblissement en $d B$ d'un pôle extrême ( 1 ou N) par rapport au pôle central M. Pour calculer $\gamma_{i m}: j=|i-m|$ avec $\beta=\beta_{c}$, affaiblissement en $\mathrm{dB}$ entre deux pôles éloignés de $\mathrm{L}$, la longueur de référence. H3 : La longueur de référence est une longueur effective $\mathrm{L}_{e}$ donnée par :

$$
2 L_{e}=\frac{1}{N} \sum_{i=1}^{N} x_{1} a_{1}
$$

\section{2 Erreur standard}

Un calcul numérique à partir des équations (3) permet de calculer l'erreur standard $\mu$ à partir des équations (1) et (7). En déplaçant le point d'observation, à $h$ constant, $\mu$ varie de 0 à $l^{\prime}$ aplomb de la source, à une valeur maximale $\mu_{M}$ pour une incidence $\pi / 4$, puis diminue. La figure 2 superpose les résultats de calculs à dix valeurs de $h(0,5<h<250)$. Dans ces limites $\mu$ est indépendant de $h$.

Cependant l'erreur maximale décroît quand h diminue en dessous de 5 : (fígure 3 ).

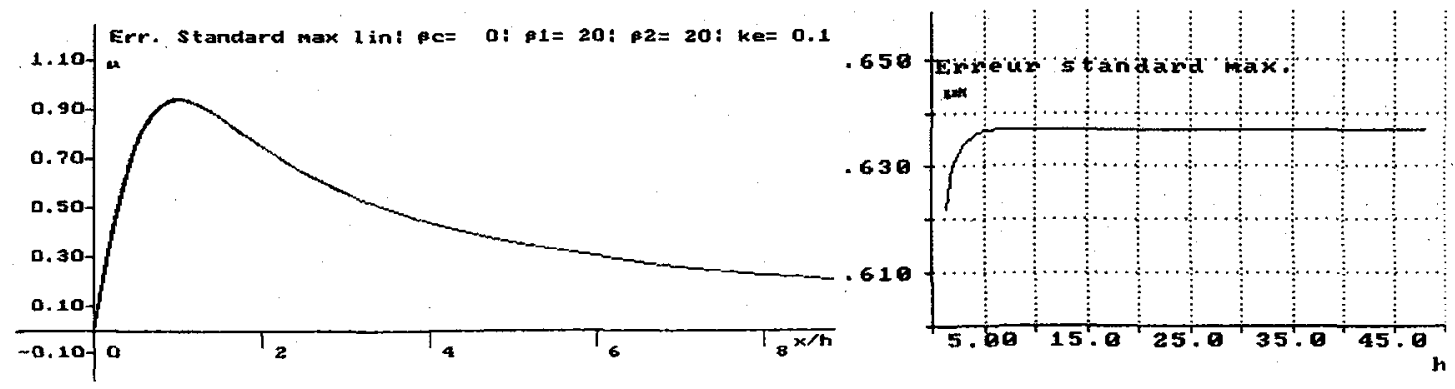

Figure $2: \mu(x / h)$ biais selon la position.

Figure $3: \mu_{x}(h)$

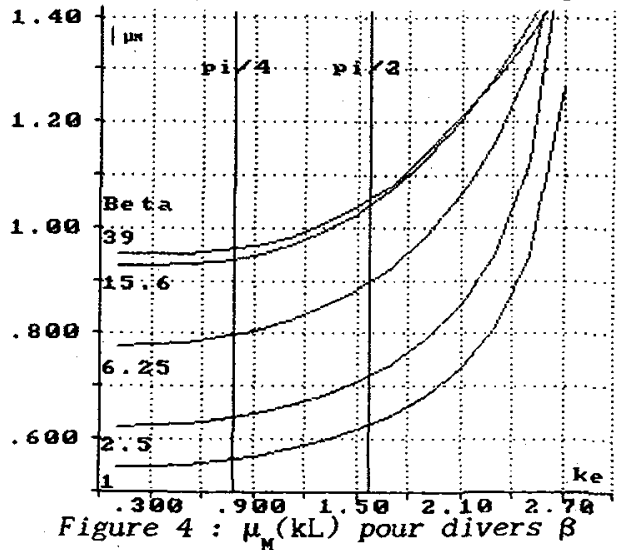

L'influence du nombre $d$ 'onde sur 1 'erreur maximale $\mu$ peut être observée, pour plusieurs valeurs de $\beta$ (figure 4). Tant que $k<\pi / 4 \mu_{\mathrm{H}}$ est sensiblement constant. $\mu_{\mathrm{H}}$ varie peu tant que $k=\mathrm{kL} \mathrm{e}_{\mathrm{e}} \pi / 2$. L'influence du nombre d'onde est donc faible quand la longueur effective totale (2Le) de la source est inférieure à une demi longueur d'onde : $\lambda / 2$.

La figure 5 donne les contours d'un blocdiagramme de $\mu \mathrm{m}$ en fonction de $\beta$ et $\beta c$, avec $k=0,1$ et $h=10$. Les valeurs de $\beta$ et $\beta_{c}$ sont données avec une progression géométrique de pas 1,5 . 


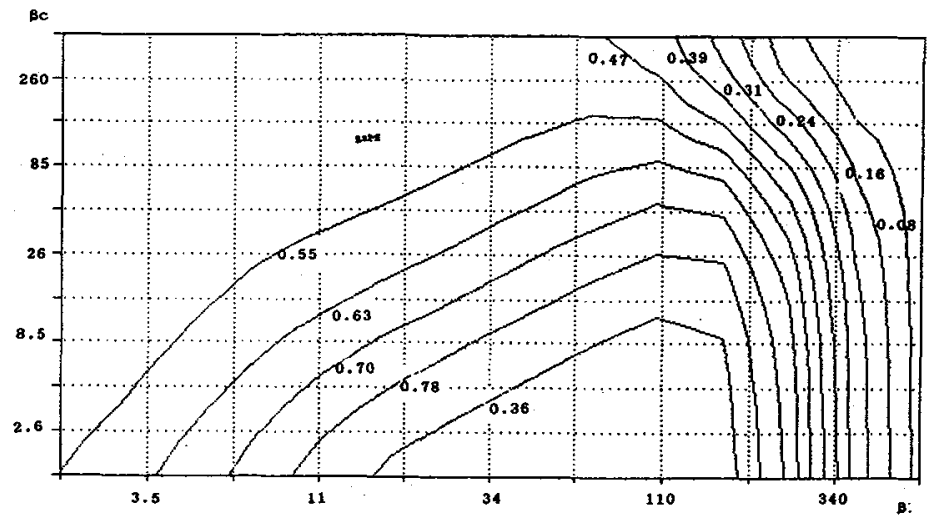

Figure 5 : Contours de $\mu \mathrm{m}$ selon $\beta$ et $\beta \mathrm{c}$

Ainsi les erreurs sont indépendantes de $h$, et aussi de $k$, si celui-ci est inférieur à $\pi / 2$. Les paramètres importants sont $\beta$ et $\beta_{c}$. Les valeurs avec $\beta$ très grand ont peu d'intérèt : ce sont des situations où un modèle avec monopôle unique serait plus représentatif. Cependant il est intéressant de vérifier que dans ce cas l'erreur tend bien vers zéro.

En excluant aussi le cas $\beta=0$, qui est celui d'une source "rectangulaire" physiquement peu vraisemblable, et en limitant les calculs à $\beta<60$, on obtient une bonne estimation de I'erreur maximale de biais $\mu_{M}$ :

$$
0,5<\mu_{Y}<1 \text { avec } 1<\beta<60 \quad L<\lambda / 2 \text { quel que soit } \beta_{c}
$$

\section{3 EXEMPLE}

Pour préciser la signification physique de (11) il est possible de considérer un jet de diamètre $D$, où pour une pulsation $\omega$ donnée, la longueur effective $L_{e}$ serait de la forme $\alpha \mathrm{D}$.

$$
\mu=\frac{\Delta \mathrm{X}}{\mathrm{D}} \cdot \frac{\mathrm{H}}{\mathrm{D}} \cdot \frac{1}{\alpha} 2<1
$$

Une mesure unique de $I$ conduit à une erreur inférieure à :

$$
\alpha^{2} \cdot \mathrm{D} / \mathrm{H}
$$

Il est envisageable d'effectuer un ensemble de mesures, en divers points, à $H$ constant. A ce moment l'écart entre les deux positions extrêmes déterminées est $2 . \Delta x$. La position de la source est, sans ambiguité, le milieu de ce segment. Comme $\mu_{M}$ est compris entre 0,5 et 1 , il est possible d'écrire :

$$
0,5<\frac{\Delta \mathrm{X}}{\mathrm{D}} \cdot \frac{\mathrm{H}}{\mathrm{D}} \cdot \frac{1}{\alpha^{2}}<1
$$

En posant $\sigma=\sqrt{\frac{2 \Delta \mathrm{X}}{\mathrm{D}} \frac{\mathrm{H}}{\mathrm{D}}}$ il apparaît possible de calculer une estimation de $\alpha$, et par conséquent, de la longueur effective de la source, à la pulsation $\omega$ :

$$
\sigma \sqrt{2}<\alpha<\sigma
$$


VI CONCLUSIONS

Dans l'hypothèse d'une source acoustique étendue avec une répartition symètrique autour d'un axe, modèlisable par une répartition symétrique de monopôles sur cet axe, il est possible de localiser le "centre" de cette source par une mesure intensimètrique 3D unique. Il existe alors un biais réauit de mesure $\varepsilon$ dont la borne supérieure est donnée par :

$$
(\varepsilon)_{\max }=1
$$

Une détermination sans biais est possible, à condition de faire des mesures en plusieurs points à la distance réduite constante $h$, avec une excursion angulaire très supérieure à $\pi / 2$. Des informations sur la longueur effective de sources sont alors disponibles.

\section{REFERENCES}

[1] S. CANDEL : La Recherche Aérospatiale No $1983 / 6$ pp 395-411

[2] D. GELY, G. ELIAS : 1er Congrès Fr. Acou. C2 T51 pp 1129-1190

[3] O. COSTE, J-C. PATRAT : 1er Congrès Fr. Acou. C2 T51 pp 895-898

[4] J-C. REBILLAT, S. RIFAI : J. Acou 3-1990 pp 263-271 